\title{
Article
}

\section{Does Physical Activity Predict Obesity-A Machine Learning and Statistical Method-Based Analysis}

\author{
Xiaolu Cheng ${ }^{1}\left(\mathbb{D}\right.$, Shuo-yu Lin ${ }^{1}\left(\mathbb{D}\right.$, Jin Liu ${ }^{2}$, Shiyong Liu ${ }^{3}$, Jun Zhang ${ }^{4}$, Peng Nie ${ }^{5} \mathbb{D}$, Bernard F. Fuemmeler ${ }^{6}$, \\ Youfa Wang ${ }^{7}$ and Hong Xue ${ }^{1, *}$
}

check for

updates

Citation: Cheng, X.; Lin, S.-y.; Liu, J.; Liu, S.; Zhang, J.; Nie, P.; Fuemmeler, B.F.; Wang, Y.; Xue, H. Does Physical Activity Predict Obesity-A Machine Learning and Statistical Method-Based Analysis. Int. J. Environ. Res. Public Health 2021, 18, 3966. https://doi.org/ $10.3390 /$ ijerph18083966

Academic Editor: Paul Tchounwou

Received: 16 February 2021

Accepted: 4 April 2021

Published: 9 April 2021

Publisher's Note: MDPI stays neutral with regard to jurisdictional claims in published maps and institutional affiliations.

Copyright: (c) 2021 by the authors. Licensee MDPI, Basel, Switzerland. This article is an open access article distributed under the terms and conditions of the Creative Commons Attribution (CC BY) license (https:/ / creativecommons.org/licenses/by/ $4.0 /)$.
1 Department of Health Administration and Policy, George Mason University, Fairfax, VA 22030, USA; xcheng4@gmu.edu (X.C.); slin26@gmu.edu (S.-y.L.)

2 Department of Biostatistics, School of Medicine, Virginia Commonwealth University, Richmond, VA 23219, USA; liuj32@vcu.edu

3 Center for Governance Studies, Beijing Normal University at Zhuhai, Zhuhai 519087, China; shiyongliu2006@gmail.com

4 Department of Physics and Engineering, Slippery Rock University of Pennsylvania, Slippery Rock, PA 16057, USA; jun.zhang@sru.edu

5 Department of Economics, School of Economics and Finance, Xi'an Jiaotong University, Xi'an 710049, China; niepeng2017@xjtu.edu.cn

6 Department of Health Behavior and Policy, School of Medicine, Virginia Commonwealth University, Richmond, VA 23219, USA; bernard.fuemmeler@vcuhealth.org

7 Global Health Institute, School of Public Health, Xi'an Jiaotong University Health Science Center, Xi'an 710049, China; youfawang@gmail.com

* Correspondence: hxue4@gmu.edu; Tel.: +1-703-993-9833

Abstract: Background: Obesity prevalence has become one of the most prominent issues in global public health. Physical activity has been recognized as a key player in the obesity epidemic. Objectives: The objectives of this study are to (1) examine the relationship between physical activity and weight status and (2) assess the performance and predictive power of a set of popular machine learning and traditional statistical methods. Methods: National Health and Nutrition Examination Survey (NHANES, 2003 to 2006) data were used. A total of 7162 participants met our inclusion criteria (3682 males and 3480 females), with average age ranging from 48.6 (normal weight) to 52.1 years old (overweight). Eleven classifying algorithms-including logistic regression, naïve Bayes, Radial Basis Function (RBF), local k-nearest neighbors (k-NN), classification via regression (CVR), random subspace, decision table, multiobjective evolutionary fuzzy classifier, random tree, J48, and multilayer perceptron-were implemented and evaluated, and they were compared with traditional logistic regression model estimates. Results: With physical activity and basic demographic status, of all methods analyzed, the random subspace classifier algorithm achieved the highest overall accuracy and area under the receiver operating characteristic (ROC) curve (AUC). The duration of vigorous-intensity activity in one week and the duration of moderate-intensity activity in one week were important attributes. In general, most algorithms showed similar performance. Logistic regression was middle-ranking in terms of overall accuracy, sensitivity, specificity, and AUC among all methods. Conclusions: Physical activity was an important factor in predicting weight status, with gender, age, and race/ethnicity being less but still essential factors associated with weight outcomes. Tailored intervention policies and programs should target the differences rooted in these demographic factors to curb the increase in the prevalence of obesity and reduce disparities among sub-demographic populations.

Keywords: physical activity; obesity; machine learning; disparity

\section{Introduction}

Over the last decade, the rapid rise in obesity prevalence has become one of the most prominent issues in global public health [1,2]. In 2015-2016, 39.6\% of U.S. adults aged 20 
and older and $18.5 \%$ of children and adolescents aged 2-19 were obese [3]. Obesity is of public health interest because excess fat would result in serious health consequences [4], including a high risk of hypertension, hypercholesterolemia, Type 2 diabetes [5], certain cancers [6], and early mortality [4]. As a result, the U.S. spends USD 315.8 billion annually treating obesity-related illness [7], and obesity is one of the top preventable causes of death in the U.S. [8].

Physical activity has been recognized as a key player in the obesity epidemic. It was found that the adherence rate to the physical activity (PA) guideline among U.S. adults remained low and unchanged between 2007 and 2016 [9]. Nationally representative data showed that in 2015, only $18 \%$ of the obese population met the PA guideline [10]. Previous evidence has suggested that a small increase in daily moderate-to-vigorous physical activity (5-10 $\mathrm{min}$ ) was associated with a lower risk of obesity [11]. It was shown that those who maintained a healthy weight were consistently more likely to engage in vigorous physical activity [12]. Additionally, physical activity interventions can benefit children and adolescents with obesity [13] and reduce obesity-related health risks [14]. However, there are also other studies suggesting a low or null correlation between PA and weight status. For example, Lauran and others, using the Behavioral Risk Factor Surveillance System (BRFSS) and the National Health and Nutrition Examination Survey (NHANES), found that from 2001 to 2009, for every 1 percentage point increase in PA prevalence, obesity prevalence was 0.11 percentage points lower after controlling social detriments of health such as poverty, unemployment, and urbanicity [15]. Using the NHANES dated from 1988 to 2006, Ruth and colleagues revealed factors other than PA could contribute more to the increase in Body mass index (BMI) over time [16]. A prior systematic review concluded that, even though the health benefit of PA has been well documented, PA alone might be a minor determinant of obesity $[17,18]$.

The relationship between physical activity and obesity remains unknown, given the mixed evidence in the extant literature. Thanks to technological advancement in recent years, machine learning (ML) has become an available powerful tool to help us identify the complex risk factors of the obesity problem. ML provides a novel way to analyze multifactorial data that can be further used to make predictions about the complex interrelationships that likely drive the risk for obesity. Comparing with statistical modeling, machine learning learns from data without relying on rules and by not only focusing on relationships between variables in a way to circumvent issues regarding overfitting, collinearity, and assumptions that are crucial in regression models. Machine learning can handle extremely large volumes of highly complicated big data much better than statistical modeling $[19,20]$. The ML techniques, however, have not always performed better in clinical settings. A systematic review showed no performance benefit of ML models over traditional logistic regression for clinical prediction models [21]. In addition, there are some hurdles existing in ML-based prediction models that undermine the usefulness of this method. For instance, a better performing ML model, in terms of some accuracy measurements, will often be in conflict with the human understanding of predictions, and there is no rule of thumb for how much performance improvement is sufficient to justify using less interpretable estimators [22]. As a result, in the present exploratory study, we aimed to: (1) examine the relationship between PA and weight status using ML techniques and objectively measured national PA data and (2) compare the findings and performance of using ML-based and traditional statistical technique-based methods in assessing the relationship between PA and weight status. We hypothesized that PA is an important predictor of whether one encounters obesity or not and that ML models could yield a better predicting power in comparison with traditional statistical methods.

\section{Materials and Methods}

\subsection{Data}

The data used were from the National Health and Nutrition Examination Survey (NHANES) [23] 2003-2004 and 2005-2006. The NHANES was conducted by the Centers 
for Disease Control and Prevention (CDC). It is a nationally representative cross-sectional survey that aims to investigate the health and nutrition statuses of Americans [23]. PA data collected by a physical activity monitor (PAM) contain objective information on the intensity and duration of common locomotion activities. Respondents wore ActiGraph AM-7164 devices [24] for seven consecutive days. The device was placed on a flexible fabric belt that fitted each individual and was worn on the right hip [25]. The advantage of using objective measurement is to reduce the recall and reporting bias commonly seen in self-reported surveys. The accelerometer in ActiGraph AM-7164 recorded the sum of the magnitude of acceleration during every one-minute epoch. The sum of device intensity values (the magnitude of acceleration) and the duration (the sum of minutes) of PAs of different intensity during the seven days were both used in the experiments.

The data were collected through both interviews and physical examinations. In this study, we used the NHANES 2003-2004 $(n=10,122)$ and 2005-2006 $(n=10,348)$ mainly because the PAM was first available in these two waves (2003-2004 and 2005-2006). The analytic sample was restricted to adults aged 20-85 for whom detailed demographic, PA, and anthropometric information (including weight and height) was available for two waves. In addition, we excluded female respondents who were pregnant at the time of the survey. The final analytical sample totaled 7162 .

\subsection{Outcome Variables}

BMI and overweight or obese weight status are the primary outcomes of this study. BMI was calculated as the respondent's weight $(\mathrm{kg})$ divided by squared height $\left(\mathrm{m}^{2}\right)$. We adopted the clinical definition of overweight and obesity from 2000 CDC growth charts-normal weight is defined as $18.5 \leq \mathrm{BMI}<25 \mathrm{~kg} / \mathrm{m}^{2}$, overweight as $25 \leq \mathrm{BMI}<30 \mathrm{~kg} / \mathrm{m}^{2}$, and obesity as BMI $\geq 30 \mathrm{~kg} / \mathrm{m}^{2}$ [26].

\subsection{Exposure Variables}

PA levels were grouped into five categories: sedentary (intensity $<100$ counts $/ \mathrm{min}$ ), light (100 $\leq$ intensity $<760$ counts / $\mathrm{min})$, lifestyle ( $760 \leq$ intensity $<2200$ counts $/ \mathrm{min})$, moderate (2200 $\leq$ intensity $<6000$ counts $/ \mathrm{min}$ ), and vigorous (intensity $\geq 6000$ counts $/ \mathrm{min}$ ) [27].

\subsection{Covariates}

Covariates in the analyses were sociodemographic variables including gender, age, race, education level, marital status, and family poverty income ratio (PIR). Specifically, race was measured through 5 racial categories: non-Hispanic white, non-Hispanic black, Mexican American, other Hispanic, and other race (including multi-racial). The education level was the highest grade or level of school the individual had completed or the highest degree the individual had received. Marital status was measured on a 6-point scale: married, widowed, divorced, separated, never married, and living with partner. The family poverty income ratio (PIR) was defined as the ratio of the individual's family income to the national poverty threshold.

\subsection{Machine Learning and Statistical Analyses}

This present study used eleven classification algorithms and traditional logistic regression to examine the relationship between PA and weight status. The eleven classification algorithms were naïve Bayes, radial basis function (RBF), local k-nearest neighbors (KNN), classification via regression (CVR), random subspace, decision table, multiobjective evolutionary fuzzy classifier, random tree, J48, and multilayer perceptron classification [28,29]. The naïve Bayes classifier is based on Bayes' theorem [30]. The conditional distribution is learned under the assumption that all attributes are mutually independent. This technique has low complexity and high scalability. However, if the independence assumption is unmet, it may not perform well. The RBF classifier [31] is a supervised learning method. It uses Gaussian radial basis function networks and normalizes all attributes. The local KNN classifier detects k-nearest neighbors of one object in the attributes space with local metric 
induction [32,33]. The class to which the object is assigned rests on the classes to which its nearest neighbors belong. Although the KNN classifier is relatively easy to understand and implement, its primary disadvantage is that it would not perform well when attributes are heterogeneous and data are imbalanced. The CVR classifier uses regression models to evaluate the class value [31]. However, the class value should be binary. Both the random subspace and J48 classifiers [34] are based on tree-like models. One attribute is tested in one node. The random subspace classifier is based on decision trees, constructing trees in randomly chosen subspaces [35]. A decision table classifier builds a decision table and makes decisions by following the rules in the table [36]. Splitting one decision table into smaller ones is relatively simple but scaling up is more difficult. Although decision trees are well suited for classification of obesity, their greedy algorithm generates an approximation of the optimal decision tree and therefore may be more computationally complex and complicated to implement [37]. The multiobjective evolutionary fuzzy classifier is a fuzzy, rule-based model to optimize two objectives [38-40]. It generates comprehensible fuzzy rules to obtain optimal solutions by maximizing the accuracy and minimizing the number of fuzzy rules, but generating these rules is time-consuming [38]. Finally, the multilayer perceptron classifier is a kind of feedforward neural network [38] with high accuracy, but the computational complexity is high and requires a large amount of data. It utilizes a backpropagation-supervised learning technique for training [41,42]. The major advantage of modeling using neural networks is that they are self-adaptive models and are capable of approximating any functional form as closely as desired [43].

\subsection{Terminology of Model Evaluation Tools and Definition}

To evaluate the predictability of eleven algorithms, we used overall accuracy, sensitivity, specificity, and the receiver operating characteristic (ROC) curve. Overall accuracy is defined as the proportion of samples that are predicted correctly. Sensitivity, known as the true positive rate, is defined as the proportion of actual positives that are correctly detected. In our study, prediction of overweight or obesity was considered as the positive. It measured the probability of the classifier to accurately detect the individuals who were at risk of being overweight (or obese). Specificity is the true negative rate, indicating the proportion of actual negative (non-overweight or non-obese) individuals who are identified by the classifiers as negative. Additionally, normal weight was considered to be the negative. The receiver operating characteristic (ROC) curve is a useful tool for organizing classifiers and visualizing their performance. It is a curve located in a two-dimensional plot where the $\mathrm{x}$ axis is the false positive rate and the $\mathrm{y}$ axis is the true positive rate. The area under the curve (AUC) value can be calculated from the ROC curve. A higher AUC value indicates a better model performance.

To evaluate the importance of each predictor, we used an information gain algorithm to rank the contribution of each predictor. Information gain is based on the concept of reduction in entropy. Entropy, in information theory, measures the amount of information that is missing before reception. It is a concave function with 0 being the smallest value and 1 being the largest in binary learning tasks and thus suitable for the present study [36]. A factor with high information gain was ranked higher because it has stronger classifying power. All analyses were conducted using WEKA or SAS.

\section{Results}

Table 1 reports the key characteristics of the study sample, including demographic, anthropometric, and physical activity features. There were 7162 participants in the sample set, $51.41 \%$ of whom were male. The individuals in the study consisted of adults from 20 to 85 years old. The mean age of the obese group (50.02) was lower than the overweight group (52.13) and higher than the healthy group (48.60). There were five racial categories: non-Hispanic white, non-Hispanic black, Mexican American, other Hispanic, and other race (including multi-racial). The education level was the highest grade or level of school the individual had completed or the highest degree the individual had received. There 
were six types of marital status: married, widowed, divorced, separated, never married, and living with partner. Only 117 individuals refused to answer this question. The family poverty income ratio (PIR) is the ratio of the individual's family income to the national poverty threshold. The lowest value is 0 , and the highest value is 5 . The mean family PIR of the overweight class was 2.74, which was higher than the other two groups. The mean value of the sum of intensity values had a positive association with group BMI. The sum of intensity values of obese groups was lower than the other two groups. The obese group also had the longest duration of sedentary activities and the shortest duration of other types of activities. For physical activity variables, the difference between the healthy and overweight groups was generally not obvious.

Table 1. Study population characteristics.

\begin{tabular}{|c|c|c|c|c|c|c|}
\hline \multirow{2}{*}{ Variables } & \multicolumn{2}{|c|}{ Normal $\left(18.5 \leq \mathrm{BMI}<25 \mathrm{~kg} / \mathrm{m}^{2}\right)$} & \multicolumn{2}{|c|}{ Overweight $\left(25 \leq \mathrm{BMI}<30 \mathrm{~kg} / \mathrm{m}^{2}\right)$} & \multicolumn{2}{|c|}{ Obese $\left(\mathrm{BMI} \geq 30 \mathrm{~kg} / \mathrm{m}^{2}\right)$} \\
\hline & Observations & Mean (\%) & Observations & Mean (\%) & Observations & Mean $(\%)$ \\
\hline \multicolumn{7}{|l|}{ Gender } \\
\hline Male & 1046 & 47.72 & 1505 & 59.65 & 1131 & 46.22 \\
\hline Female & 1146 & 52.28 & 1018 & 40.35 & 1316 & 53.78 \\
\hline Age & 2192 & 48.60 & 2523 & 52.13 & 2447 & 50.02 \\
\hline \multicolumn{7}{|l|}{ Race } \\
\hline Non-Hispanic White & 806 & 36.77 & 857 & 33.97 & 708 & 28.93 \\
\hline Non-Hispanic Black & 334 & 15.24 & 368 & 14.59 & 508 & 20.76 \\
\hline Mexican American & 420 & 19.16 & 593 & 23.50 & 606 & 24.77 \\
\hline Other Race, Including Multi-Racial & 342 & 15.60 & 396 & 15.70 & 410 & 16.76 \\
\hline Other Hispanic & 290 & 13.23 & 309 & 12.25 & 215 & 8.79 \\
\hline \multicolumn{7}{|l|}{ Education Level } \\
\hline Less than 9th Grade & 702 & 32.03 & 917 & 36.35 & 862 & 35.23 \\
\hline $\begin{array}{l}\text { 9-11th Grade (Includes 12th } \\
\text { Grade with No Diploma) }\end{array}$ & 251 & 11.45 & 280 & 11.1 & 292 & 11.93 \\
\hline $\begin{array}{c}\text { High School Grad/GED or } \\
\text { Equivalent }\end{array}$ & 347 & 15.83 & 438 & 17.36 & 465 & 19 \\
\hline Some College or AA Degree & 344 & 15.69 & 369 & 14.63 & 400 & 16.35 \\
\hline College Graduate or Above & 451 & 20.57 & 421 & 16.69 & 341 & 13.94 \\
\hline Refused & 95 & 4.33 & 95 & 3.77 & 87 & 3.56 \\
\hline Do Not Know & 1 & 0.05 & 1 & 0.04 & 0 & 0 \\
\hline \multicolumn{7}{|l|}{ Marital Status } \\
\hline Married & 807 & 36.82 & 1016 & 40.27 & 903 & 36.90 \\
\hline Widowed & 445 & 20.30 & 514 & 20.37 & 485 & 19.82 \\
\hline Divorced & 305 & 13.91 & 331 & 13.12 & 356 & 14.55 \\
\hline Separated & 148 & 6.75 & 203 & 8.05 & 219 & 8.95 \\
\hline Never Married & 327 & 14.92 & 276 & 10.94 & 319 & 13.04 \\
\hline Living with Partner & 126 & 5.75 & 143 & 5.67 & 122 & 4.99 \\
\hline Refused & 34 & 1.55 & 40 & 1.59 & 43 & 1.76 \\
\hline Family PIR & 2192 & 2.62 & 2523 & 2.74 & 2447 & 2.60 \\
\hline Sum Intensity Value & 2192 & $1,584,527.52$ & 2523 & $1,562,816.99$ & 2447 & $1,298,389.77$ \\
\hline \multicolumn{7}{|l|}{$\begin{array}{l}\text { Duration of Different Activity } \\
\text { Intensity Levels (in Minutes) }\end{array}$} \\
\hline Sedentary & 2192 & 7988.89 & 2523 & 7945.10 & 2447 & 8146.58 \\
\hline Light & 2192 & 1468.27 & 2523 & 1498.67 & 2447 & 1407.09 \\
\hline Lifestyle & 2192 & 500.84 & 2523 & 522.37 & 2447 & 450.55 \\
\hline Moderate & 2192 & 112.70 & 2523 & 106.91 & 2447 & 73.21 \\
\hline Vigorous & 2192 & 7.45 & 2523 & 4.63 & 2447 & 1.93 \\
\hline
\end{tabular}

\subsection{Relationship between PA and Overweight/Obesity}

We first predicted whether an individual was overweight/obese based on PA levels. The individuals were divided into two groups based on their BMIs: normal and abnormal (including both overweight and obese individuals). Table 2 represents the classification performance results. The random subspace classifier had the highest overall accuracy of $70.01 \%$. The sensitivity of the J48 classifier was $72.9 \%$. The highest specificity was $57.3 \%$ for the random subspace classifier, while the lowest specificity was $35.6 \%$ for the random tree model. The mean percentages of overall accuracy, sensitivity, and specificity of the eleven 
classifiers were $62.37 \%, 70.89 \%$, and $49.70 \%$, respectively. The sensitivities for all models were much higher than the corresponding specificities.

Table 2. Evaluations of accuracy, sensitivity, and specificity of 11 prediction models of overweightness and obesity.

\begin{tabular}{|c|c|c|c|c|c|c|c|c|c|c|c|c|}
\hline Method & $\begin{array}{c}\text { Logistic } \\
\text { Regression }\end{array}$ & $\begin{array}{l}\text { Naïve } \\
\text { Bayes }\end{array}$ & $\mathrm{RBF}^{\dagger}$ & $\begin{array}{c}\text { Local } \\
\text { KNN }^{+}\end{array}$ & $\mathrm{CVR}^{+}$ & $\begin{array}{l}\text { Random } \\
\text { Subspace }\end{array}$ & $\begin{array}{c}\text { Decision } \\
\text { Table }\end{array}$ & Multiobject & $\begin{array}{c}\text { Random } \\
\text { Tree }\end{array}$ & $\mathrm{J} 48 \ddagger$ & $\begin{array}{l}\text { Multilayer } \\
\text { Perceptron }\end{array}$ & $\begin{array}{l}\text { Mean Value } \\
\text { of } 11 \text { Models }\end{array}$ \\
\hline Acc & $69.4 \%$ & $69.11 \%$ & $69.5 \%$ & $69.63 \%$ & $69.9 \%$ & $70.01 \%$ & $69.59 \%$ & $69.37 \%$ & $60.71 \%$ & $68.70 \%$ & $68.65 \%$ & $62.37 \%$ \\
\hline Sensitivity & $70.0 \%$ & $70.8 \%$ & $70.2 \%$ & $70.7 \%$ & $70.9 \%$ & $70.6 \%$ & $70.1 \%$ & $70.0 \%$ & $71.6 \%$ & $72.9 \%$ & $72.0 \%$ & $70.89 \%$ \\
\hline Specificity & $51.6 \%$ & $48.1 \%$ & $51.6 \%$ & $52.1 \%$ & $54.4 \%$ & $57.3 \%$ & $51.4 \%$ & $49.5 \%$ & $35.6 \%$ & $47.9 \%$ & $47.2 \%$ & $49.70 \%$ \\
\hline
\end{tabular}

Notes: ${ }^{+} \mathrm{RBF}=$ radial basis function; $\mathrm{KNN}=$ local $\mathrm{k}$-nearest neighbors; $\mathrm{CVR}=$ classification via regression; random subspace; ${ }^{\ddagger} \mathrm{J} 48=\mathrm{J} 48$ is an algorithm to generate decision trees.

The sensitivity of the logistic regression method was slightly lower than the mean value, while the specificity was slightly higher than the mean value. The random tree performed worst in overall accuracy and specificity, although it achieved a higher sensitivity compared with the mean sensitivity value. In general, all the methods have similar overall accuracy, sensitivity, and specificity. As shown in the ROC curves in Figure 1, the random subspace classifier produced the highest result with $63.3 \%$ area under the ROC curve (AUC). The other four classifiers, namely, RBF, CVR, decision table, and multilayer perceptron, also achieved marginally over $62 \%$ AUC. Naïve Bayes and J48 classifiers proved average in AUC, with approximately $60 \%$. The AUC values of local KNN and random tree classifiers were slightly higher than $53 \%$. The AUC value from the multiobject classifier was the lowest of $51.2 \%$.

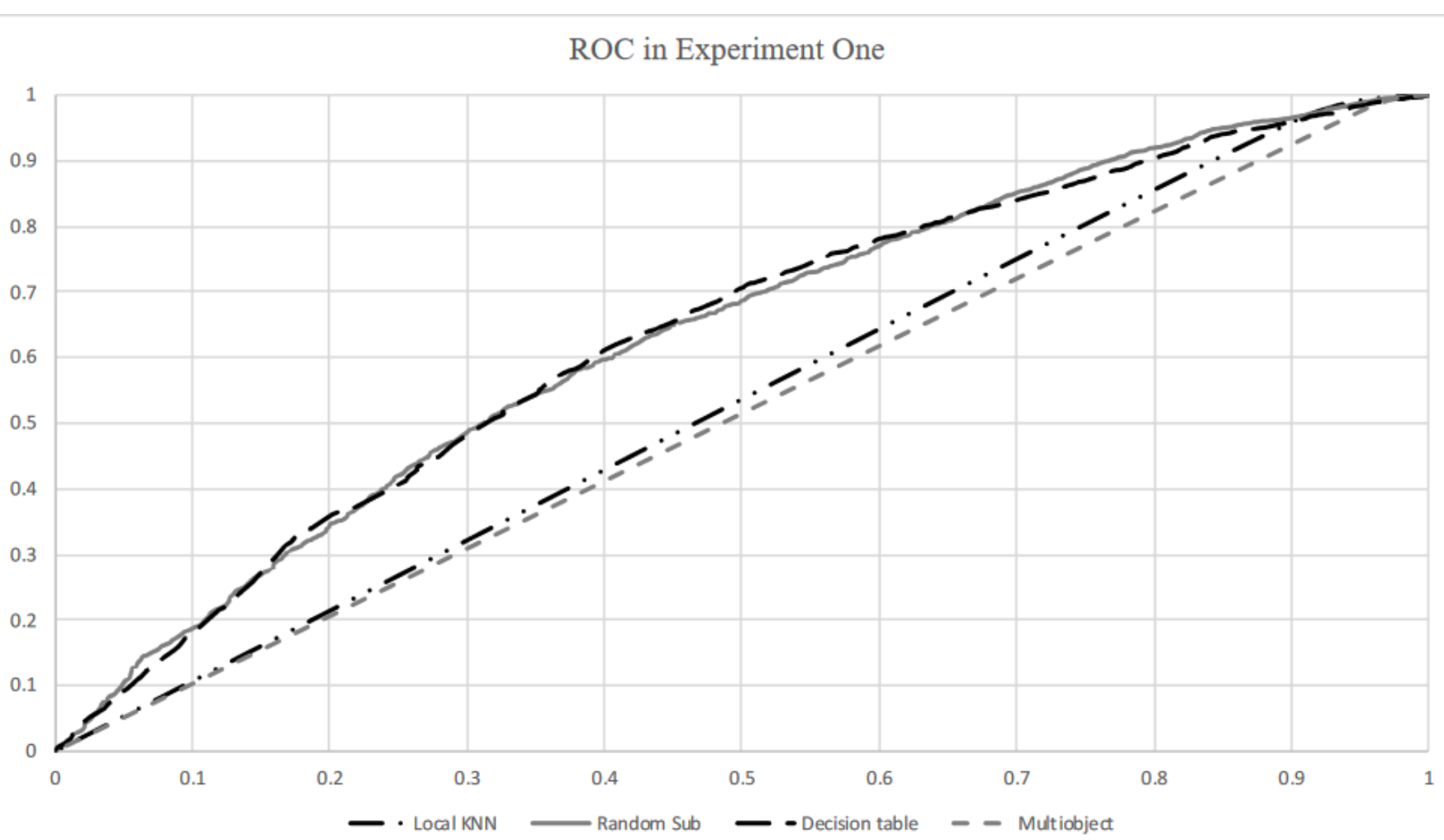

Figure 1. Receiver operating characteristic (ROC) curves for local KNN, random subspace, decision table, and multiobject algorithms.

\subsection{Relationship between PA and Obesity Only}

We then predicted whether an individual was obese based on PA levels. The individuals were labeled as not obese and obese. The results of classifying are shown in Table 3 . The mean overall accuracy was $57.69 \%$, which was lower than that of Table $2(62.37 \%)$. Likewise, the mean sensitivity was $48.55 \%$, which was much lower than that of Table 2 (70.89\%). 
Nonetheless, the mean specificity was $68.75 \%$, which was significantly higher than that of Table $2(49.70 \%)$. The random subspace classifier had the highest overall accuracy and sensitivity. Compared with other methods, naïve Bayes had the highest specificity (76.7\%). However, the overall accuracy $(49.64 \%)$ of the naïve Bayes classifier was significantly lower than that of the other ten classifiers. Regarding overall accuracy, the logistic regression model performed slightly worse than the other algorithms, excluding naïve Bayes, random tree, and J48, all of which performed lower than the logistic regression classifier. Both the sensitivity $(49.4 \%)$ and specificity $(66.5 \%)$ of logistic regression were slightly higher than the mean values of all eleven models. Table 3 also demonstrates that for all of the classifiers, specificities were higher than sensitivities. The sensitivities of six algorithms (including logistic regression, naïve Bayes, multiobject, random tree, J48, multilayer perceptron) were lower than $50 \%$. The highest sensitivity obtained from random subspace was only $56.8 \%$.

Table 3. Evaluations of accuracy, sensitivity, and specificity of 11 prediction models of obesity.

\begin{tabular}{|c|c|c|c|c|c|c|c|c|c|c|c|c|}
\hline Method & $\begin{array}{c}\text { Logistic } \\
\text { Regression }\end{array}$ & $\begin{array}{l}\text { Naïve } \\
\text { Bayes }\end{array}$ & $\mathbf{R B F}^{\dagger}$ & $\begin{array}{l}\text { Local } \\
\text { KNN }\end{array}$ & CVR & $\begin{array}{l}\text { Random } \\
\text { Subspace }\end{array}$ & $\begin{array}{c}\text { Decision } \\
\text { Table }\end{array}$ & Multiobject & $\begin{array}{c}\text { Random } \\
\text { Tree }\end{array}$ & $\mathrm{J} 48 \ddagger$ & $\begin{array}{l}\text { Multilayer } \\
\text { Perceptron }\end{array}$ & $\begin{array}{l}\text { Mean Value } \\
\text { of } 11 \text { Models }\end{array}$ \\
\hline Accuracy & $65.78 \%$ & $49.64 \%$ & $66.20 \%$ & $65.92 \%$ & $65.89 \%$ & $67.03 \%$ & $66.32 \%$ & $65.78 \%$ & $58.42 \%$ & $63.66 \%$ & $64.48 \%$ & $57.69 \%$ \\
\hline Sensitivity & $49.4 \%$ & $38.6 \%$ & $52.7 \%$ & $50.3 \%$ & $50.3 \%$ & $56.8 \%$ & $52.4 \%$ & $49.1 \%$ & $39.5 \%$ & $45.8 \%$ & $49.2 \%$ & $48.55 \%$ \\
\hline Specificity & $66.5 \%$ & $76.7 \%$ & $67.1 \%$ & $68.1 \%$ & $67.2 \%$ & $68.0 \%$ & $67.9 \%$ & $66.3 \%$ & $68.7 \%$ & $69.9 \%$ & $69.9 \%$ & $68.75 \%$ \\
\hline
\end{tabular}

Notes: ${ }^{\dagger} \mathrm{RBF}=$ radial basis function; $\mathrm{KNN}=$ local $\mathrm{k}$-nearest neighbors; $\mathrm{CVR}=$ classification via regression; random subspace; ${ }^{\ddagger} \mathrm{J} 48=\mathrm{J} 48$ is an algorithm to generate decision trees.

Figure 2 illustrates the ROC curves of the four classifiers that achieved the two highest AUC values and the two lowest AUC values. Both random subspace and CVR classifiers achieved $64.3 \%$ AUC values. However, RBF, decision table, and multilayer perceptron classifiers performed slightly worse than the random subspace classifier. The naïve Bayes classifier achieved $62 \%$ AUC in obesity prediction. The multiobject classifier still had the lowest AUC of 51\%. In general, all classifiers produced similar AUC values to those of Table 2.

\section{ROC in Experiment Two}

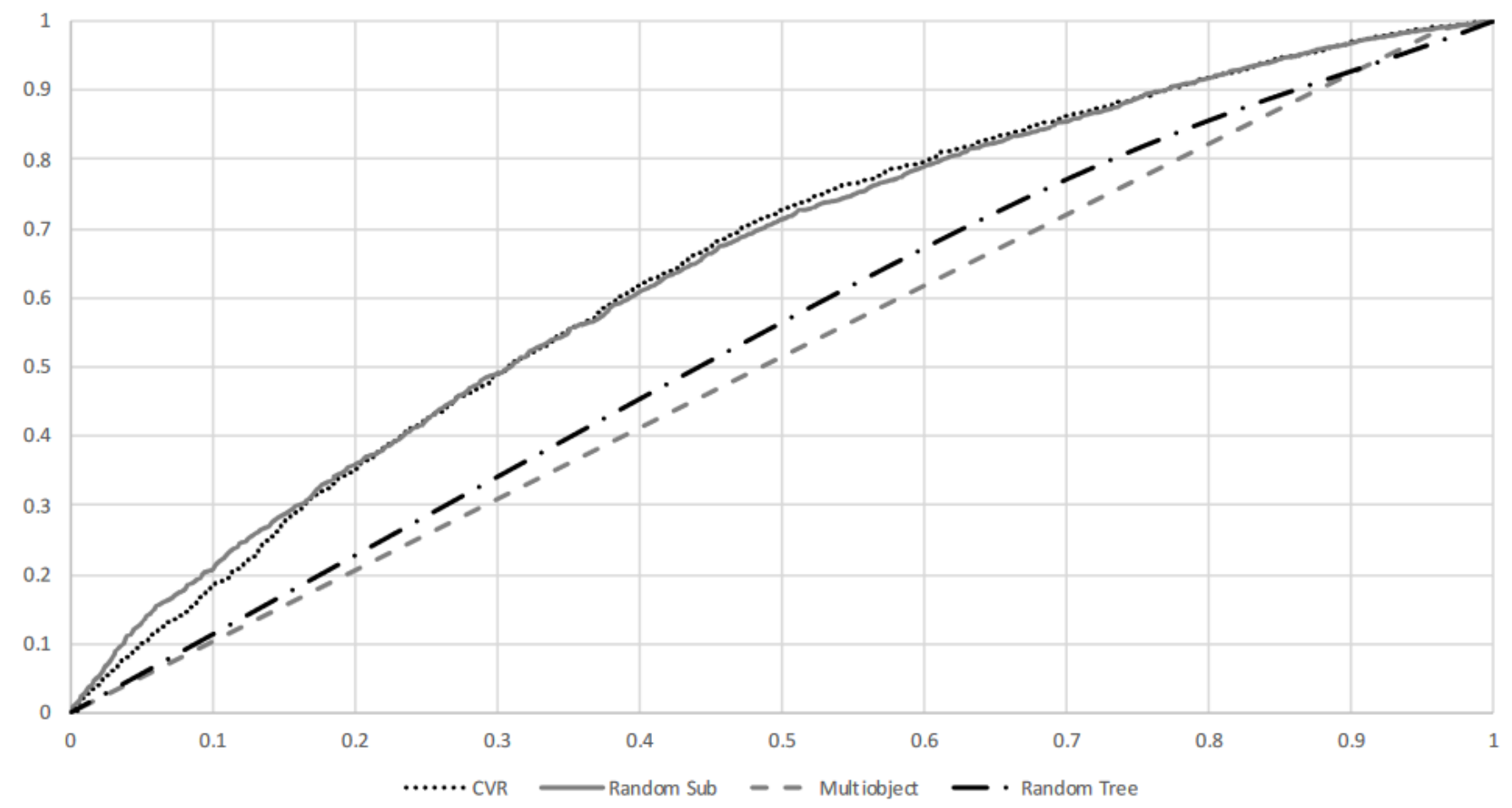

Figure 2. ROC curves for CVR, random subspace, random tree, and multiobject algorithms. 


\subsection{Importance of PA in Predicting Weight Status}

We further checked variable importance by ranking all features involved in predictive models. All features were ranked by an information gain ranking filter. One Rule is a classifier that only generates one rule for each feature. The importance of a feature was evaluated by the accuracy of the rule. Table 4 shows the feature ranking results.

Table 4. Ranked feature importance in predicting weight status based on information gain.

\begin{tabular}{ccc}
\hline Rank & Feature Meaning & Contribution \\
\hline 1 & Duration of moderate-intensity activity in one week & 0.0211 \\
2 & Duration of vigorous-intensity activity in one week & 0.0140 \\
3 & Age & 0.0137 \\
4 & The sum of the intensity value recorded by the & 0.0133 \\
5 & physical activity monitor in one week & 0.0110 \\
6 & Race/Ethnicity & 0.0053 \\
7 & Duration of sedentary-intensity activity in one week & 0.0045 \\
8 & Duration of lifestyle-intensity activity in one week & 0.0042 \\
9 & Duration of light-intensity activity in one week & 0.0040 \\
10 & Gender & 0.0027 \\
11 & Education level & 0 \\
12 & Poverty income ratio (PIR) & 0 \\
\hline
\end{tabular}

Duration of vigorous-intensity activity in one week and duration of moderate-intensity activity in one week were ranked top among 12 features with contributions of 0.0211 and 0.014 , respectively. Age was ranked No.3 with a 0.0137 contribution. Gender, race/ethnicity, and socioeconomic status (SES) (marital status, education level, and poverty income ratio), even though they contributed to the prediction model, had less predictive power, ranking among the bottom.

\section{Discussion}

This study is the first study in the field to assess adulthood overweight/obese risks using objectively measured PA data and advanced ML techniques. Our results indicate that PA (especially moderate to vigorous intensity) was a key risk factor in predicting overweight and obesity. In addition, duration of moderate-intensity activity and duration of vigorous-intensity activity ranked higher than the sum of the intensity value recorded by the physical activity monitor, duration of sedentary-intensity activity, duration of lifestyle-intensity activity, and duration of light-intensity activity. The results imply that high-intensity physical activity is more important than low-intensity physical activity and overall physical activity intensity. This finding aligns with our hypothesis and empirical evidence. Physical activity increases a person's energy expenditure and helps individuals maintain their energy balance or even lose weight as long as energy consumption is not compensated by calorie intake [44]. Demographic features played a less but still critical role in predicting weight status as our ML and traditional statistical analyses suggested. This finding is in line with previous studies [15,45]. Underlying disparities associated with race/ethnicity including food environment, healthcare access, social environment, and building environment are important factors that predict the risk of obesity through complex known and unknown pathways [46]. This may provide guidance for obesity control.

We compared the performance of different ML algorithms in predicting weight status and found that the random subspace algorithm had a weak superiority over the other ten models. However, the logistic regression model performed slightly worse than the random subspace and several other algorithms. The random subspace classifier produced the highest overall accuracy and AUC among the eleven classifiers. It also produced the highest specificity for prediction of normal weight and the highest sensitivity for prediction of obesity. The sensitivity of the J48 classifier in normal weight prediction was $2.3 \%$ higher than the sensitivity of the random subspace classifier. The specificities of the J 48 classifier 
in both normal weight and obesity predictions were also higher than the random subspace classifier. The overall accuracies of the J48 classifier were lower than those of the random subspace classifier. Furthermore, its sensitivity was $11.0 \%$ lower than the sensitivity of the random subspace classifier in prediction of obesity. Overall, the random subspace classifier outperformed the J48 classifier. Regarding obesity prediction, the specificity produced by the naïve Bayes classifier was $8.7 \%$ higher than that of the random subspace classifier. However, it performed worse than the other algorithms regarding overall accuracy and sensitivity.

Our results are comparable to existing studies. For instance, Jie et al. [47] reviewed 927 studies and provided no evidence of the superior performance of advanced machine learning methods over the logistic regression method for clinical predictions. Similarly, Tozlu et al. [48] used logistic regression and four other machine learning methods (adaptive boosting, ANN, RF, and SVM) to identify high infarction risk. They concluded that logistic regression performed as well as the other four machine learning approaches. In addition, Tozlu et al. [48] compared the performances of several machine learning models to predict the effect of labor induction on the occurrence of cesarean section and found that the logistic regression model produced a similar performance to the RF one. Therefore, advanced machine learning methods with high computational complexity are not always necessary in obesity prediction. Due to the well-understood theoretical and computational background, the logistic regression model is preferable when predicting obesity.

Some limitations of this study should be noted: First, ActiGraph AM-7164 is not waterproof, and thus some activities such as swimming were not captured. Thus, the collected data for some individuals who performed aquatic activities are not trustable. The device may not have been able to accurately detect upper-body exercises, such as lifting dumbbells. Second, this study only examined weight status but not body composition, which is planned for our future research.

\section{Conclusions}

This study was conducted in a large population-based sample and thus the results reflect the average relationship between physical activity and BMI. Weight loss intervention trials testing the addition of physical activity to diet modification have shown the importance of physical activity at weight-loss maintenance. The findings herein do not speak to the types and level of physical activity needed for individuals trying to lose weight.

In sum, the random subspace algorithm had a weak superiority over the other ten models including logistic regression. Although physical activity was a crucial predictor of weight outcomes, demographic characteristics including gender, age, and race/ethnicity were also important factors associated with weight outcomes. Tailored intervention policies and programs should target the differences rooted in these demographic factors to curb the increase in the prevalence of obesity and reduce disparities among subdemographic populations.

Author Contributions: Conceptualization, H.X. and X.C.; methodology, H.X., X.C., S.-y.L.; software, X.C., S.-y.L.; validation, H.X., X.C., S.-y.L., J.L., S.L., J.Z., P.N., B.F.F., Y.W.; formal analysis, H.X., X.C., S.-y.L.; investigation, H.X., X.C., S.-y.L., J.L., S.L., J.Z., P.N., B.F.F., Y.W.; data curation, H.X., X.C., S.-y.L.; writing-original draft preparation, H.X., X.C., S.-y.L.; writing-review and editing, H.X., X.C., S.-y.L., J.L., S.L., J.Z., P.N., B.F.F., Y.W.; visualization, H.X., X.C., S.-y.L.; supervision, H.X.; project administration, H.X., X.C. All authors have read and agreed to the published version of the manuscript.

Funding: This research received no external funding.

Institutional Review Board Statement: Not applicable.

Data Availability Statement: Data sharing not applicable.

Conflicts of Interest: The authors declare no conflict of interest. 


\section{References}

1. Levesque, R.J. Obesity and overweight. In Encyclopedia of Adolescence; Springer: Cham, Switzerland, 2018; pp. $2561-2565$.

2. Wang, Y.; Lim, H. The Global Childhood Obesity Epidemic and the Association between Socio-Economic Status and Childhood Obesity; Taylor \& Francis: Abingdon, UK, 2012.

3. Hales, C.M.; Carroll, M.D.; Fryar, C.D.; Ogden, C.L. Prevalence of Obesity among Adults and Youth: United States, 2015-2016; NCHS Data Brief, No 288; National Center for Health Statistics: Hyattsville, MD, USA, 2017.

4. Peeters, A.; Barendregt, J.J.; Willekens, F.; MacKenbach, J.P.; Al Mamun, A.; Bonneux, L. Obesity in Adulthood and Its Consequences for Life Expectancy: A Life-Table Analysis. Ann. Intern. Med. 2003, 138, 24-32. [CrossRef]

5. Thompson, D.; Edelsberg, J.; Colditz, G.A.; Bird, A.P.; Oster, G. Lifetime health and economic consequences of obesity. Arch. Intern. Med. 1999, 159, 2177-2183. [CrossRef]

6. $\quad$ Bhaskaran, K.; Douglas, I.; Forbes, H.; Dos-Santos-Silva, I.; Leon, D.A.; Smeeth, L. Body-mass index and risk of 22 specific cancers: A population-based cohort study of $5 \cdot 24$ million UK adults. Lancet 2014, 384, 755-765. [CrossRef]

7. Cawley, J. An economy of scales: A selective review of obesity's economic causes, consequences, and solutions. J. Health Econ. 2015, 43, 244-268. [CrossRef]

8. Danaei, G.; Ding, E.L.; Mozaffarian, D.; Taylor, B.; Rehm, J.; Murray, C.J.; Ezzati, M. The preventable causes of death in the United States: Comparative risk assessment of dietary, lifestyle, and metabolic risk factors. PLoS Med. 2009, 6, e1000058. [CrossRef] [PubMed]

9. Du, Y.; Liu, B.; Sun, Y.; Snetselaar, L.G.; Wallace, R.B.; Bao, W. Trends in Adherence to the Physical Activity Guidelines for Americans for Aerobic Activity and Time Spent on Sedentary Behavior Among US Adults, 2007 to 2016. JAMA Netw. Open 2019, 2, e197597. [CrossRef] [PubMed]

10. Churilla, J.R.; Johnson, T.M.; Richardson, M.R.; Williams, B.D.; Rariden, B.S.; Boltz, A.J. Mode of physical activity participation by body mass index: 2015 behavioural risk factor surveillance system. Res. Sports Med. 2018, 26, 147-157. [CrossRef] [PubMed]

11. Maher, C.A.; Mire, E.; Harrington, D.M.; Staiano, A.E.; Katzmarzyk, P.T. The independent and combined associations of physical activity and sedentary behavior with obesity in adults: NHANES 2003-06. Obesity 2013, 21, E730-E737. [CrossRef]

12. Spees, C.K.; Scott, J.M.; Taylor, C.A. Differences in amounts and types of physical activity by obesity status in US adults. Am. J. Health Behav. 2012, 36, 56-65. [CrossRef]

13. Martin, A.; Booth, J.N.; Laird, Y.; Sproule, J.; Reilly, J.J.; Saunders, D.H. Physical activity, diet and other behavioural interventions for improving cognition and school achievement in children and adolescents with obesity or overweight. Cochrane Database Syst. Rev. 2018, 1, CD009728. [CrossRef]

14. Fogelholm, M. Physical activity, fitness and fatness: Relations to mortality, morbidity and disease risk factors. A systematic review. Obes. Rev. 2010, 11, 202-221. [CrossRef]

15. Dwyer-Lindgren, L.; Freedman, G.; Engell, R.E.; Fleming, T.D.; Lim, S.S.; Murray, C.J.; Mokdad, A.H. Prevalence of physical activity and obesity in US counties, 2001-2011: A road map for action. Popul. Health Metrics 2013, 11, 7. [CrossRef] [PubMed]

16. Brown, R.E.; Sharma, A.M.; Ardern, C.I.; Mirdamadi, P.; Mirdamadi, P.; Kuk, J.L. Secular differences in the association between caloric intake, macronutrient intake, and physical activity with obesity. Obes. Res. Clin. Pract. 2016, 10, 243-255. [CrossRef] [PubMed]

17. Wilks, D.C.; Besson, H.; Lindroos, A.K.; Ekelund, U. Objectively measured physical activity and obesity prevention in children, adolescents and adults: A systematic review of prospective studies. Obes. Rev. 2010, 12, e119-e129. [CrossRef]

18. Chin, S.-H.; Kahathuduwa, C.N.; Binks, M. Physical activity and obesity: What we know and what we need to know*. Obes. Rev. 2016, 17, 1226-1244. [CrossRef]

19. Ngiam, K.Y.; Khor, I.W. Big data and machine learning algorithms for health-care delivery. Lancet Oncol. 2019, 20 , e262-e273. [CrossRef]

20. Desai, R.J.; Wang, S.V.; Vaduganathan, M.; Evers, T.; Schneeweiss, S. Comparison of Machine Learning Methods with Traditional Models for Use of Administrative Claims with Electronic Medical Records to Predict Heart Failure Outcomes. JAMA Netw. Open 2020, 3, e1918962. [CrossRef]

21. Christodoulou, E.; Ma, J.; Collins, G.S.; Steyerberg, E.W.; Verbakel, J.Y.; Van Calster, B. A systematic review shows no performance benefit of machine learning over logistic regression for clinical prediction models. J. Clin. Epidemiol. 2019, 110, 12-22. [CrossRef]

22. Doupe, P.; Faghmous, J.; Basu, S. Machine Learning for Health Services Researchers. Value Health 2019, 22, 808-815. [CrossRef]

23. Johnson, C.L.; Paulose-Ram, R.; Ogden, C.L.; Carroll, M.D.; Kruszan-Moran, D.; Dohrmann, S.M.; Curtin, L.R. National health and nutrition examination survey: Analytic guidelines, 1999-2010. Vital Health Stat 2 2013, 161, 1-24.

24. John, D.; Freedson, P. ActiGraph and Actical physical activity monitors: A peek under the hood. Med. Sci. Sports Exerc. 2012, 44 (Suppl. 1), S86. [CrossRef]

25. Centers for Disease Control and Prevention (CDC); National Center for Health Statistics (NCHS). National Health and Nutrition Examination Survey Questionnaire (or Examination Protocol, or Laboratory Protocol). 2021. Available online: http:/ /www.cdc. gov/nchs/nhanes.htm (accessed on 12 December 2020).

26. Kuczmarski, R.J.; Ogden, C.L.; Guo, S.S.; Grummer-Strawn, L.M.; Flegal, K.M.; Mei, Z.; Wei, R.; Curtin, L.R.; Roche, A.F.; Johnson, C.L. 2000 CDC Growth Charts for the United States: Methods and development. Vital Health Stat. 2002, 11, 1-190.

27. Wanner, M.; Richard, A.; Martin, B.; Faeh, D.; Rohrmann, S. Associations between self-reported and objectively measured physical activity, sedentary behavior and overweight/obesity in NHANES 2003-2006. Int. J. Obes. 2017, 41, 186-193. [CrossRef] [PubMed] 
28. Cook, D.; Dixon, P.; Duckworth, W.; Kaiser, M.; Koehler, K.; Meeker, W.; Stephenson, W. Binary Response and Logistic Regression Analysis; Part of the Iowa State University NSF/ILI project Beyond Traditional Statistical Methods; Iowa State University: Ames, IA, USA, 2001; pp. 1-23.

29. Lewis, R.J. An introduction to classification and regression tree (CART) analysis. In Proceedings of the Annual Meeting of the Society for Academic Emergency Medicine, San Francisco, CA, USA, 22-25 May 2000.

30. Maron, M.E. Automatic indexing: An experimental inquiry. J. ACM (JACM) 1961, 8, 404-417. [CrossRef]

31. Frank, E. Fully Supervised Training of Gaussian Radial Basis Function Networks in WEKA; University of Waikato: Hamilton, New Zealand, 2014.

32. Altman, N. An introduction to kernel and nearest-neighbor nonparametric regression. Am. Stat. 1992, 46, 175-185.

33. Frank, E.; Wang, Y.; Inglis, S.; Holmes, G.; Witten, I.H. Using model trees for classification. Mach. Learn. 1998, 32, 63-76. [CrossRef]

34. Quinlan, J.R. C4. 5: Programs for Machine Learning; Elsevier: Amsterdam, The Netherlands, 2014.

35. Ho, T.K. The random subspace method for constructing decision forests. IEEE Trans. Pattern Anal. Mach. Intell. 1998, $20,832-844$.

36. Lei, S. A feature selection method based on information gain and genetic algorithm. In Proceedings of the 2012 International Conference on Computer Science and Electronics Engineering, Hangzhou, China, 23-25 March 2012; Volume 2.

37. DeGregory, K.W.; Kuiper, P.; DeSilvio, T.; Pleuss, J.D.; Miller, R.; Roginski, J.W.; Fisher, C.B.; Harness, D.; Viswanath, S.; Heymsfield, S.B.; et al. A review of machine learning in obesity. Obes. Rev. 2018, 19, 668-685. [CrossRef] [PubMed]

38. Jiménez, F.; Sánchez, G.; Juárez, J.M. Multi-objective evolutionary algorithms for fuzzy classification in survival prediction. Artif. Intell. Med. 2014, 60, 197-219. [CrossRef] [PubMed]

39. Nam, D.; Park, C.H. Multiobjective simulated annealing: A comparative study to evolutionary algorithms. Int. J. Fuzzy Syst. 2000, 2,87-97.

40. Fazzolari, M.; Alcala, R.; Nojima, Y.; Ishibuchi, H.; Herrera, F. A review of the application of multiobjective evolutionary fuzzy systems: Current status and further directions. IEEE Trans. Fuzzy Syst. 2012, 21, 45-65. [CrossRef]

41. White, B.J. Availability of categories and contrast-effects in judgment. Am. J. Psychol. 1964, 77, 231-239. [CrossRef] [PubMed]

42. Rumelhart, D.H.; Williams, R.J. Learning internal representations by error propagation. Parallel Distrib. Process. 1986, 1, 1-33.

43. Zhang, G.P. Neural networks for classification: A survey. IEEE Trans. Syst. Man Cybern. Part C Appl. Rev. 2000, $30,451-462$. [CrossRef]

44. Strasser, B. Physical activity in obesity and metabolic syndrome. Ann. N. Y. Acad. Sci. 2012, 1281, 141-159. [CrossRef]

45. Paeratakul, S.; Lovejoy, J.; Ryan, D.; Bray, G. The relation of gender, race and socioeconomic status to obesity and obesity comorbidities in a sample of US adults. Int. J. Obes. 2002, 26, 1205-1210. [CrossRef]

46. Kirby, J.B.; Liang, L.; Chen, H.-J.; Wang, Y. Race, Place, and Obesity: The Complex Relationships Among Community Racial/Ethnic Composition, Individual Race/Ethnicity, and Obesity in the United States. Am. J. Public Health 2012, 102, 1572-1578. [CrossRef]

47. Ladanie, A.; Speich, B.; Briel, M.; Sclafani, F.; Bucher, H.C.; Agarwal, A.; Ioannidis, J.P.; Pereira, T.V.; Kasenda, B.; Hemkens, L.G. Single pivotal trials with few corroborating characteristics were used for FDA approval of cancer therapies. J. Clin. Epidemiol. 2019, 114, 49-59. [CrossRef]

48. Tozlu, C.; Ozenne, B.; Cho, T.; Nighoghossian, N.; Mikkelsen, I.K.; Derex, L.; Hermier, M.; Pedraza, S.; Fiehler, J.; Østergaard, L.; et al . Comparison of classification methods for tissue outcome after ischaemic stroke. Eur. J. Neurosci. 2019, 50, 3590-3598. [CrossRef] [PubMed] 